\title{
Editorial: promotion for physical activity in elderly
}

\author{
Michael Sagiv
}

Received: 27 July 2008 / Accepted: 30 July 2008 / Published online: 16 August 2008

(C) EGREPA 2008

Keywords Aging · Cardiovascular · Work capacity · Exercise $\cdot$ Pulmonary function

Aging considerations Aging is associated with decreases in aerobic capacity, cardiac function, and insulin sensitivity as well as alterations in myocardial substrate metabolism. Myocardial functional changes with cardiac complications in sedentary elderly include decline in maximum heart rate, stroke volume, and contractility, and an increase in peripheral vascular resistance. The changes in structure and function of the cardiovascular system result in $\mathrm{VO}_{2}$ max decline, which is the best single indicator of physical working capacity. Whether maximal cardiac output actually decreases depends on many interactive factors. First, the primary aging process, which has a genetic component, occurs in the absence of disease and independent of lifestyle [11]. If oxygen transport is indeed limited by maximal cardiac output, this may be calculated as the product of maximal heart rate, arteriovenous oxygen difference, and maximal stroke volume. Some studies have shown correlation between $\mathrm{VO}_{2}$ max and muscle mass [3]. Mitochondrial density was found to be lower in skeletal muscle of none active individuals, which could further diminish capacity for endurance work [5].

In humans, maximal heart rate peaks at around 10 years of age, and decreases by approximately 1 beat per minute, per

\section{Sagiv}

Life sciences Department,

Zinman College of Physical Education and Sport Sciences,

Wingate 42902, Israel

\section{Sagiv $(\bowtie)$}

Division Sports Medicine and Rehabilitation, Zinman College,

Wingate 42902, Israel

e-mail: sagiv@wincol.ac.il year. This suggests that maximal heart rate does not adapt to chronic exercise [10]. The mechanism(s) underlying the decreased maximal heart rate is unknown. The changes may be attributed to the heart itself rather than to neural input. An example for the input ability of the nervous system in the elderly and patients with coronary artery disease is the manifestation of ventricular arrhythmias. Other investigations suggested that there is an age-related decline in maximal heart rate which could be associated with decreased sympathetic drive, due to diminished response to adrenergic regulation of the heart and circulation [6].

Inactivity is associated with alterations in body composition such that there is an increase in percentage of body fat and a concomitant decline in lean body mass. Thus, significant loss in maximal force production takes place with inactivity.

The decline in muscle strength and mass due to inactivity [9] has been linked to reduction in metabolic function. Dynamic exercise increases aerobic metabolism of the exercising skeletal muscles in proportion to the mass of muscles and intensity of exertion involved. Skeletal muscle atrophy is often considered a hallmark of aging and physical inactivity. This deficit has profound implications for the regeneration of ATP in the muscles. Oxidative capacity declines in some skeletal muscles with advancing age [4].

Training effect considerations The role of exercise in maintaining health has been investigated in both human and animal studies. Exercise training in the elderly was associated with health benefits and specifically with decreased cardiovascular mortality in two large observational studies [7]. Recently, [1], it was suggested that following training the mechanism most likely to be involved is a change in the cardiac autonomic balance producing an increase, or a relative dominance, of the vagal component. It has been long known 
that exercise training reduces resting and sub-maximal heart rate. Several cardiac changes accompany the normal aging process, including prolongation of excitation - contraction and relaxation, and increase in after-load, increased vascular and myocardial stiffness, and decreased catecholamine sensitivity [8].

Alterations in left ventricular structure and function are a well-described and accepted component of the response to physical conditioning. This is true if elderly or younger subjects are engaged in similar endurance training. Both age groups demonstrated cardiac changes previously documented to occur with exercise training, including lower heart rates, larger ventricular cavities, lower wall stresses, and higher passive/active phases ratio.

The training effect on cardiopulmonary function during sub-maximal exercise of a fixed absolute work rate is similar for younger and elderly.

Endurance exercise training in elderly decreases resting and sub-maximal exercise heart rate, systolic and diastolic blood pressure while stroke volume increased. Marked changes are notable in the elderly during peak effort in which stroke volume, cardiac output, contractility, and oxygen uptake are increased, while total peripheral resistance, systolic and diastolic blood pressure decreased, thus lowering after-load which in turn facilitate left ventricular systolic and diastolic function.

That efficiency of breathing is improved by reducing in lactic acid levels and increased maximal ventilation and an augmentation of respiratory capacity in the muscle with increases in the ability to oxidize pyruvate, fatty acids, and ketones. As a result of increases in the levels of the enzymes of the malate-asparate shuttle, there is also an enhancement of the capability for mitochondrial oxidation of the reducing equivalents generated in the cytoplasm during glycolysis. The rise in muscle respiratory capacity results from an increase in muscle mitochondria and an alteration in mitochondrial composition, making skeletal muscle mitochondria more like heart mitochondria in their enzyme pattern [2]. The changes in muscle oxidative potential may play a major role in the subjects' capacity to perform sub-maximal work.

In conclusion, physical activity is the cornerstone of a good health program. A structured or prescribed aerobic program, regularly followed, has been shown to reduce the incidence of coronary disease, increase the quality of living, and probably reduce the incidence of further heart attacks in the elderly.
New data suggest an increased life expectancy for those who exercise regularly. Ideally, a program to increase vitality and quality of life should be started during childhood. These data indicate that the skeletal muscle of elderly, cardiovascular system and pulmonary function retain a high degree of trainability, with much of the improvement occurring peripherally, just as in younger individuals.

\section{References}

1. Eynon N, Sagiv M, Amir O, Ben-Sira D, Goldhammer E, Amir R (2008) The effect of long-term beta-adrenergic receptor blockade on the oxygen delivery and extraction relationship in patients with coronary artery disease. J Cardiopulm Rehabil Prev 28:189-194

2. Fernström M, Bakkman L, Tonkonogi M, Shabalina IG, Rozhdestvenskaya Z, Mattsson CM et al (2007) Reduced efficiency, but increased fat oxidation, in mitochondria from human skeletal muscle after 24-h ultraendurance exercise. J Appl Physiol 102:1844 1849 doi:10.1152/japplphysiol.01173.2006

3. Fleg JL, Morrell CH, Bos AG, Brant LJ, Talbot LA, Wright JG et al (2005) Accelerated longitudinal decline of aerobic capacity in healthy older adults. Circulation 112:674-682 doi:10.1161/ CIRCULATIONAHA.105.545459

4. Forrest KY, Zmuda JM, Cauley JA (2007) Patterns and correlates of muscle strength loss in older women. Gerontology 53:140-147 doi:10.1159/000097979

5. Kallinen M, Suominen H, Vuolteenaho O, Alen M (1998) Effort tolerance in elderly women with different physical activity backgrounds. Med Sci Sports Exerc 30:170-176 doi:10.1097/00005768199801000-00024

6. Lakatta EG (1993) Deficient neuroendocrine regulation of the cardiovascular system with advancing age in healthy humans. Circulation 87:631-636

7. Laukkanen JA, Kurl S, Salonen R, Rauramaa R, Salonen JT (2004) The predictive value of cardiorespiratory fitness for cardiovascular events in men with various risk profiles: a prospective populationbased cohort study. Eur Heart J 25:1428-1437 doi:10.1016/j.ehj.2004. 06.013

8. McGuire DK, Levine BD, Williamson JW, Snell PG, Blomqvist CG, Saltin B et al (2001) A 30-year follow-up of the Dallas Bedrest and Training Study: I. Effect of age on the cardiovascular response to exercise. Circulation 104:1350-1357 doi:10.1161/hc3701.096099

9. Miller SL, Wolfe RR (2008) The danger of weight loss in the elderly. J Nutr Health Aging 12:487-491

10. Sagiv M, Goldhammer E, Ben-Sira D, Amir R (2007) What maintains energy supply at peak aerobic exercise in trained and untrained older men? Gerontology 53:357-361 doi:10.1159/000104898

11. Soto PF, Herrero P, Schechtman KB, Waggoner AD, Baumstark JM, Ehsani AA, Gropler RJ (2008) Exercise training impacts myocardial metabolism of older individuals in a gender-specific manner. Am J Physiol Heart Circ Physiol 295:H842-H850 